\title{
D-2-Hydroxyglutarate does not mimic all the IDH mutation effects, in particular the reduced etoposide- triggered apoptosis mediated by an alteration in mitochondrial NADH
}

\author{
K Oizel ${ }^{1,2}$, C Gratas ${ }^{1,2,3}$, A Nadaradjane ${ }^{1,2}$, L Oliver ${ }^{1,2,3}$, FM Vallette ${ }^{1,2,4}$ and C Pecqueur ${ }^{*, 1,2}$
}

Somatic mutations in isocitrate dehydrogenase (IDH)-1 and -2 have recently been described in glioma. This mutation leads to a neomorphic enzymatic activity as the conversion of isocitrate to alpha ketoglutarate $(\alpha \mathrm{KG})$ is replaced by the conversion of $\alpha \mathrm{KG}$ to $D$-2-hydroxyglutarate $(D-2 H G)$ with NADPH oxidation. It has been suggested that this oncometabolite D-2HG via inhibition of $\alpha$ KG-dioxygenases is involved in multiple functions such as epigenetic modifications or hypoxia responses. The present study is aimed at deciphering how the mutant IDH can affect cancer pathogenesis, in particular with respect to its associated oncometabolite D-2HG. We show that the overexpression of mutant IDH in glioma cells or treatment with D-2HG triggered an increase in cell proliferation. However, although mutant IDH reduced cell sensitivity to the apoptotic inducer etoposide, D-2HG exhibited no effect on apoptosis. Instead, we found that the apoptotic effect was mediated through the mitochondrial NADH pool reduction and could be inhibited by oxamate. These data show that besides D-2HG production, mutant IDH affects other crucial metabolite pools. These observations lead to a better understanding of the biology of IDH mutations in gliomas and their response to therapy.

Cell Death and Disease (2015) 6, e1704; doi:10.1038/cddis.2015.13; published online 26 March 2015

Gliomas are the most common type of human brain tumors and can be classified based on clinical and pathological criteria in four grades. The grade IV glioma, commonly known as glioblastoma multiforme (GBM), is the most invasive form and has a dismal prognosis with $<5 \%$ patient survival at 5 years. These GBM can develop de novo (primary GBM) or through the progression from low-grade tumors (secondary GBM). Although these two types of GBM are histologically similar, primary and secondary GBM exhibit distinct genetic patterns. A recent integrated genome analysis of human GBM shows that $12 \%$ of these tumors have a mutation in the gene encoding isocitrate dehydrogenase 1 (IDH1) and to a lesser extent in IDH2 gene. ${ }^{1}$ This mutation is present in $>90 \%$ secondary GBMs, whereas it is present in $<5 \%$ primary GBMs. ${ }^{2}$ Mutations in IDH1 and IDH2 have also been identified in acute myeloid leukemia $(\mathrm{AML})^{3}$ and chondrosarcomas. ${ }^{4}$ The occurrence of IDH mutations predicts a significantly longer survival for patients affected by GBM or grade III gliomas. ${ }^{1,2}$ Whether this difference is driven by IDH mutations or reflects other fundamental biological differences between primary and secondary GBM is, as yet, unclear. For example, the prognostic significance of IDH mutations may be secondary to their prevalence among younger patients, as age is a well-known prognostic factor in gliomas. ${ }^{5}$ In AML, the prognostic significance of IDH mutations is more ambiguous.
Several studies have reported that IDH mutations do not affect the prognosis in AML, whereas other studies have found that IDH mutations are associated with an increased or decreased risk of relapse when compared with IDH wild-type patients. ${ }^{6,7}$

The human genome has five IDH genes coding for three different IDH isoforms, the activities of which depend on either nicotinamide adenine dinucleotide (NAD+) for IDH3 or nicotinamide adenine dinucleotide phosphate (NADP+) for IDH1 and IDH2. Both IDH2 and IDH3 are located in the mitochondria where they participate in the TCA cycle, whereas IDH1 is mostly cytosolic. ${ }^{8}$ To date, all reported mutations are located in the IDH1 and IDH2 genes and result in an amino-acid substitution at residues located in the enzymatic active site, respectively, R132 for IDH1 and R140 or R172 for IDH2. This mutation disrupts the normal enzymatic function of IDH, that is, the conversion of isocitrate to alpha ketoglutarate $(a \mathrm{KG})$ with the concomitant production of NADPH. Instead, mutant IDH displays a neomorphic activity converting aKG into D-2-hydroxyglutarate (D-2HG), although reducing $\mathrm{NADPH} .{ }^{9}$ As a result, mutant IDH may alter the redox state of cells, modulate the activity of metabolic and epigenetic tumor suppressor enzymes that use aKG as a co-substrate. ${ }^{10}$ Loss of IDH function may also alter normal mitochondrial function and promote a metabolic switch in cancer cells to glycolysis. $^{11,12}$

\footnotetext{
${ }^{1}$ CRCNA - INSERM UMR 892 - CNRS UMR 6299, Nantes F44007, France; ${ }^{2}$ Faculté de Médecine, Université de Nantes, Nantes F44007, France; ${ }^{3}$ Centre HospitalierUniversitaire (CHU) de Nantes, Nantes F44093, France and ${ }^{4}$ Institut de Cancérologie de l'Ouest - René Gauducheau, St Herblain F44805, France

*Corresponding author: C Pecqueur, CRCNA - INSERM UMR 892 - CNRS UMR 6299, 8 quai Moncousu, Nantes F44007, France. Tel: +332 2808 0325; Fax: +33 22808 0204; E-mail: claire.pecqueur@univ-nantes.fr

Abbreviations: $\alpha \mathrm{KG}$, alpha ketoglutarate; AAT, aspartate aminotransferase; D-2HG, D-2-hydroxyglutarate; ECAR, extracellular acidification rate; ETC, electron transport chain; ETO, etoposide; GBM, glioblastoma multiforme; Gln, glutamine; Glu, glutamate; IDH, isocitrate dehydrogenase; OCR, oxygen consumption rate Received 16.9.14; revised 20.12.14; accepted 07.1.15; Edited by A Finazzi-Agrò
} 
Mutant IDH is widely believed to have the ability to transform cells by modulating aKG-dependent enzymes. D-2HG and aKG are structurally similar suggesting that $\mathrm{D}-2 \mathrm{HG}$ may act as a competitive inhibitor of aKG-dioxygenases including prolyl hydroxylase involved in HIF-1a stability, histone demethylases and the Ten-Eleven Translocation (TET) family of 5-methylcytosine hydroxylases involved in epigenetic modifications of DNA. ${ }^{13,14}$ In fact, IDH mutations lead to numerous metabolic abnormalities besides D-2HG production. Deciphering the relative importance of either $\mathrm{D}-2 \mathrm{HG}$ production, aKG or NADPH reduction in cancer pathogenesis remains to be determined. In this paper, we show that mutant IDH increases cell proliferation and reduces etoposide (ETO)induced cell death through different metabolic pathways. Although cell proliferation changes are mediated through $\mathrm{D}-2 \mathrm{HG}$, alteration in the mitochondrial NADH pool is involved in the response to apoptosis.

\section{Results}

Glioma cell lines overexpressing IDH1 ${ }^{\mathrm{R} 132}$ exhibit reduced NADP production and increased cell proliferation. To date, the lack of mutant IDH glioma cell lines has been an issue in the study of the pathogenic role of IDH mutations. To address this, we stably transfected a human glioma cell line, U251, with vectors encoding the wild-type or the mutant form of IDH1 $\left(\mathrm{IDH} 1^{\mathrm{R} 132}\right.$; Figure 1a). IDH1 was detected by western blot in cytoplasmic lysate. In order to determine whether $\mathrm{IDH} 1^{\mathrm{R} 132}$ overexpression was associated with the neomorphic enzymatic activity, the NADPH level was measured in these cells (Figure 1b). As expected, lysate from IDH1-overexpressing cells exhibited higher levels of IDH activity as compared with $\mathrm{IDH}_{1}{ }^{\mathrm{R} 132}$. Note that the level of NADPH detected in control cells was similar to the level observed in mutant IDHexpressing cells. Addition of either aKG or D-2HG to the cell medium did not affect the total cellular NADPH level (Figure 1c). In order to study how these mutations can affect cancer pathogenesis, cell proliferation and cell death response to different stimuli were measured. An increased proliferation and an increased ability to form colonies when cells were plated at very low density were detected in cells overexpressing IDH1 $1^{\mathrm{R} 132}$ (Figures 1d and e). To determine whether D-2HG had a role in this increased proliferation, cells were cultured for 6 days in the presence of aKG,

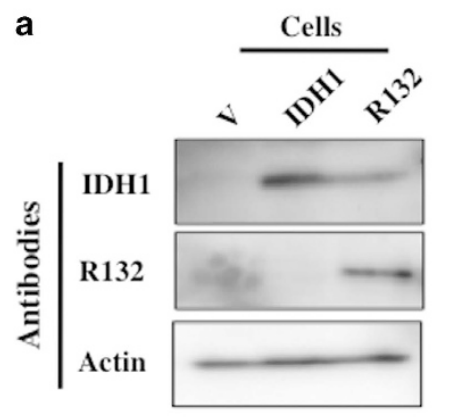

b

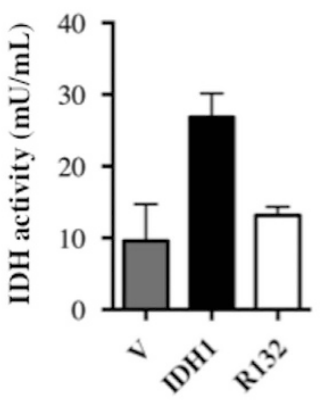

C

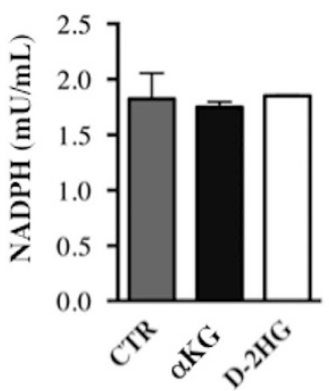

d

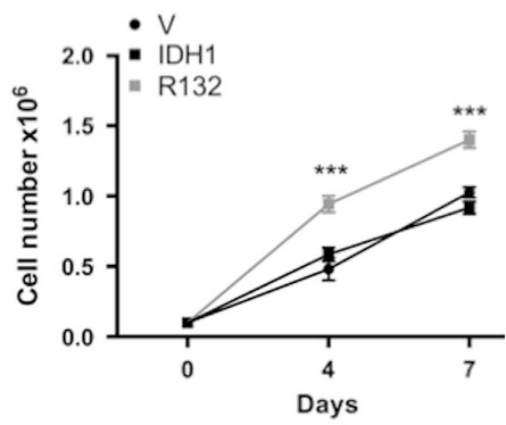

e

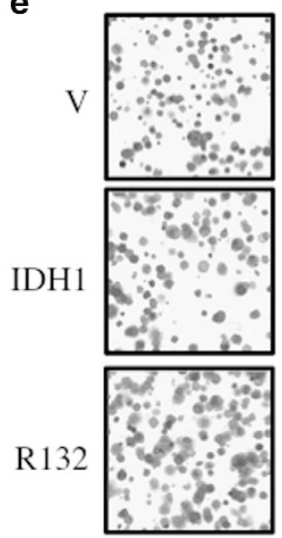

f

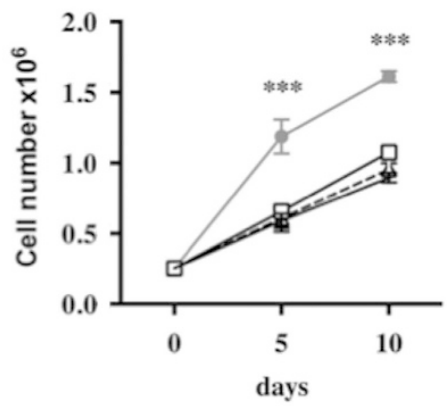

- D-2HG

$\Delta \alpha \mathrm{KG}$

dmKG

v CTR

Figure 1 (a) Expression of wild-type or mutant IDH1 isoforms in stable overexpressing cells was confirmed by immunoblotting using whole lysates ( $40 \mu \mathrm{g})$. Actin was used as a loading control. (b) IDH activity of cells overexpressing wild-type or mutant IDH1 isoforms. Cells $\left(1 \times 10^{6}\right)$ were plated, lysed the next day and subsequently assayed for their IDH activity. (c) NADPH production in cells treated for 6 days with $\alpha \mathrm{KG}(3 \mathrm{mM})$ or D-2HG $(3 \mathrm{mM})$ as in $\mathbf{b}$. (d) Proliferation of wild-type and mutant IDH1-overexpressing cells. Cells were plated at $1 \times 10^{5}$ cells and counted 3 and 7 days later using trypan blue staining. (e) The ability of forming colonies of wild-type and mutant IDH1-overexpressing cells. Cells were plated at 500 cells per well then fixed and stained with violet crystal 1 week later. (f) Proliferation of cells treated with $\alpha \mathrm{KG}(3 \mathrm{mM})$, dimethyl- $\alpha \mathrm{KG}(3 \mathrm{mM})$ and $\mathrm{HG}(3 \mathrm{mM})$. Cells were plated at $1 \times 10^{5}$ cells and counted 5 and 10 days later using trypan blue staining. Results are expressed as the mean \pm S.E.M. of three experiments performed in triplicate. V, empty vector expressing cells; IDH1, wild-type IDH1-expressing cells; R132, IDH1 ${ }^{\mathrm{R} 132}$-expressing cells transfected. ${ }^{* \star} P<0.01$ and ${ }^{\star \star \star} P<0.001$ 
a cell-permeant form of $a \mathrm{KG}$, dimethyl $a \mathrm{KG}$ (dmKG) or D-2HG. As expected, $a K G$ and dmKG had no effect on cell proliferation, whereas, similar to the overexpression of $\mathrm{IDH} 1^{\mathrm{R} 132}$, D-2HG increased significantly cell proliferation compared with the control or cells treated with the different forms of aKG (Figure 1f).

$\mathrm{IDH} 1^{\mathrm{R} 132}$ reduces ETO-induced apoptosis. Cell death was measured at different time points after irradiation (5 Gy), ETO $(50 \mu \mathrm{g} / \mathrm{ml})$, TRAIL $(50 \mathrm{ng} / \mathrm{ml})$, FasL $(60 \mathrm{ng} / \mathrm{ml})$ or cisplatin $(15 \mu \mathrm{g} / \mathrm{ml})$ treatment in U251 cells. All treatments were associated with a significant death and activation of caspase-3 (Table 1). However, the optimal time point of cell death induction varied from $6 \mathrm{~h}$ with TRAIL to $24 \mathrm{~h}$ with ETO, Cisplatin, and FASL treatments, and to $72 \mathrm{~h}$ with irradiation. Next, sensitivity to cell death was analyzed in $\mathrm{IDH} 1$ - and IDH1 ${ }^{\mathrm{R} 132}$-overexpressing cells. For most treatments, overexpression of IDH1 ${ }^{\mathrm{R} 132}$ did not affect caspase-3 activity (Figure 2a). However, although addition of ETO caused a high caspase- 3 activation in control and wild-type IDH1-overexpressing cells, activation of caspase-3 was significantly reduced in $\mathrm{IDH}_{1}{ }^{\mathrm{R} 132}$-overexpressing cells. These results were confirmed by FACs analysis, which showed that the percentage of propidium iodide-stained cells after ETO exposure was lower in IDH1 ${ }^{\mathrm{R} 132}$ cells compared with the control and IDH1 cells (Figure 2b). During apoptosis, the integrity of the mitochondrial outer membrane is compromised, a process called mitochondrial outer membrane permeabilization (MOMP). In order to determine whether IDH1 or IDH1 ${ }^{\mathrm{R} 132}$ expression was affecting the MOMP, we measured the mitochondrial membrane potential $\Delta \Psi \mathrm{m}$ in our cells using $\mathrm{JC}-1$ staining. $\Delta \psi_{\mathrm{m}}$ was reduced in a timedependent manner after ETO exposure as indicated by the decrease of the red/green ratio (Figure 2c). Of note, the median of healthy cells red fluorescence was significantly higher in IDH1 ${ }^{\mathrm{R} 132}$ cells compared with IDH1 cells $(845 \pm 75$ versus $655 \pm 56 ; n=6 ; P<0.05)$, suggesting a mitochondrial hyperpolarization of $\mathrm{IDH}_{1}{ }^{\mathrm{R} 132}$ cells. Sensitivity to ETO was then tested in other glioma cell lines overexpressing either IDH1 or IDH1 ${ }^{\mathrm{R} 132}$, LN18 and T98 (Figures 2d and e). In both cells lines, IDH1 $1^{\mathrm{R} 132}$ overexpression was associated with reduced ETO-induced cell apoptosis.

Mutant IDH2 decreases sensitivity to ETO but D-2HG has no effect. To determine whether mutant IDH2 triggered similar effects on cell death, U251 cells were transfected with vector, IDH2, IDH2 ${ }^{\mathrm{R} 140}$ and $\mathrm{IDH} 2^{\mathrm{R} 172}$. Western blot analysis showed that IDH2 proteins were expressed in the mitochondria and not in the cytosol (Figure 3a). Similarly to $\mathrm{IDH}_{1}{ }^{\mathrm{R} 132}$, mutant IDH2-expressing cells exhibited decreased NADPH consumption corresponding to its neomorphic activity (Figure 3b). Interestingly, caspase-3 activation following ETO treatment was also significantly reduced (Figure $3 \mathrm{c}$ ). Altogether, these results showed that all mutants IDH, independently of their respective subcellular localization, reduced $\mathrm{ETO}$-induced cell death.

To determine whether D-2HG could be involved in this phenotype, U251 cells were treated with $3 \mathrm{mM}$ or $10 \mathrm{mM}$ of D-2HG for 6 days before ETO treatment. However, none of the pretreatments affected ETO-induced apoptosis (Figure $3 \mathrm{~d}$ and data not shown). Thus, D-2HG does not fully recapitulate the mutant IDH phenotypes as exogenous D-2HG is able to increase cell proliferation but does not affect cell sensitivity to ETO.

Reduced mitochondrial spare capacity with $\mathrm{IDH} 1^{\mathrm{R} 132}$ but not with D-2HG. To better understand how IDH1 ${ }^{\mathrm{R} 132}$ affects apoptosis, several parameters were analyzed. IDH mutation can alter protein expression through epigenetic modifications, redox homeostasis and metabolism of glucose, glutamine (GIn) and fatty acids. As an aberrant methylation status of Bax and Bcl2 has been shown to be associated with

Table 1 Cell death was monitored in U251 after irradiation (5 Gy), ETO (50 $\mu \mathrm{g} / \mathrm{ml})$, TRAIL (50 ng/ml), FasL ( $60 \mathrm{ng} / \mathrm{ml})$ or cisplatin (15 $\mu \mathrm{g} / \mathrm{ml})$ treatment after 5, 24,48 and $72 \mathrm{~h}$ by FACS analysis using propidium iodide incorporation and caspase- 3 activation

\begin{tabular}{|c|c|c|c|c|c|}
\hline & CTR & $6 \mathrm{~h}$ & $24 \mathrm{~h}$ & $48 \mathrm{~h}$ & $72 \mathrm{~h}$ \\
\hline \multicolumn{6}{|l|}{ Etoposide } \\
\hline$\%$ Dead cells & $2.6 \pm 0.8(n=3)$ & NA & $28 \pm 2(n=4)$ & $28 \pm 3(n=4)$ & NA \\
\hline DEVDase activity & $3.3 \pm 0.3(n=3)$ & $37 \pm 11(n=4)$ & $204 \pm 18(n=4)$ & $128 \pm 11(n=4)$ & NA \\
\hline \multicolumn{6}{|l|}{ Irradiation } \\
\hline$\%$ Dead cells & $2.6 \pm 0.8(n=3)$ & NA & $6 \pm 2(n=3)$ & $8 \pm 1(n=3)$ & $15 \pm 1(n=5)$ \\
\hline DEVDase activity & $3.3 \pm 0.3(n=3)$ & NA & $65 \pm 25(n=3)$ & NA & $143 \pm 13(n=5)$ \\
\hline \multicolumn{6}{|l|}{$T R A / L$} \\
\hline$\%$ Dead cells & $2.6 \pm 0.8(n=3)$ & $22 \pm 3(n=4)$ & $6.4 \pm 1(n=3)$ & NA & NA \\
\hline DEVDase activity & $3.3 \pm 0.3(n=3)$ & $107 \pm 3(n=4)$ & $7 \pm 0.3(n=3)$ & NA & NA \\
\hline \multicolumn{6}{|l|}{ FASL } \\
\hline$\%$ Dead cells & $2.6 \pm 0.8(n=3)$ & $10 \pm 1(n=3)$ & $20 \pm 3(n=3)$ & NA & NA \\
\hline DEVDase activity & $3.3 \pm 0.3(n=3)$ & $38 \pm 8(n=3)$ & $115 \pm 3(n=3)$ & $51 \pm 13(n=3)$ & NA \\
\hline \multicolumn{6}{|l|}{ Cisplatin } \\
\hline$\%$ Dead cells & $2.6 \pm 0.8(n=3)$ & NA & $16 \pm 2(n=3)$ & NA & NA \\
\hline DEVDase activity & $3.3 \pm 0.3(n=3)$ & NA & $139 \pm 18(n=3)$ & NA & NA \\
\hline
\end{tabular}


a

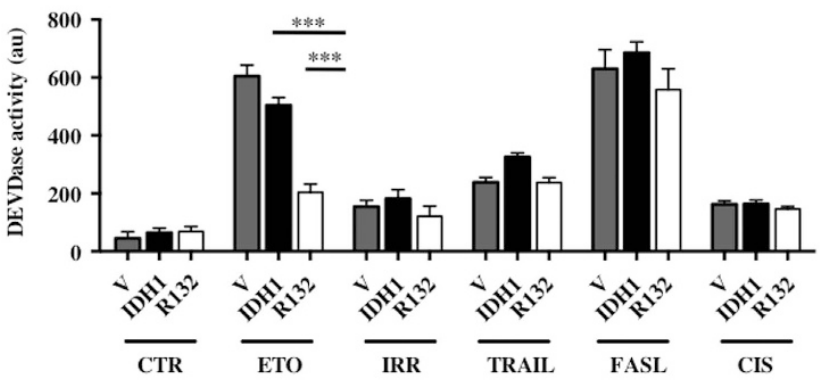

C

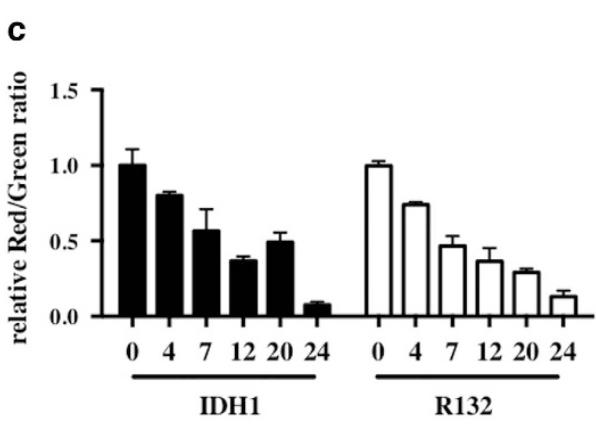

b

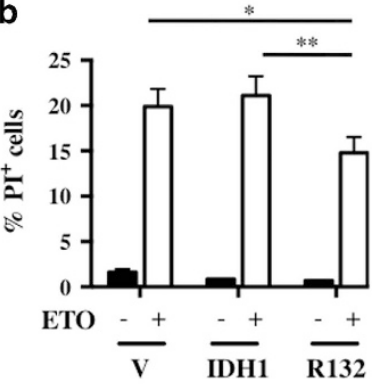

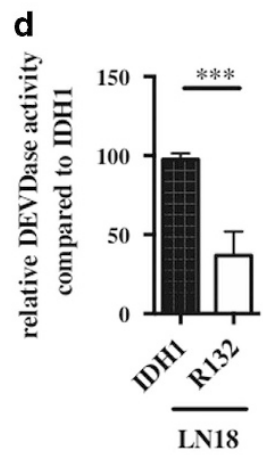

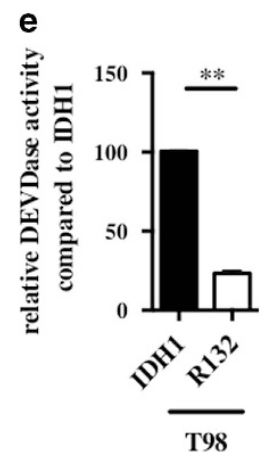

Figure 2 (a) Caspase 3 activation was determined with DEVDase activity assay in stable transfected U251 cells expressing empty vector, wild-type and mutant IDH1 isoforms after induction of apoptosis. Cells were plated at $5 \times 10^{5}$ cells and treated the next day with different inducers of cell apoptosis. Cellular extracts were prepared from untreated cells (CTR), $5 \mathrm{~h}$ after treatment with TRAIL ( $50 \mathrm{ng} / \mathrm{ml}), 24 \mathrm{~h}$ after ETO $(50 \mu \mathrm{g} / \mathrm{ml})$, FASL $(60 \mathrm{ng} / \mathrm{ml})$ or cisplatin (CIS) $(15 \mu \mathrm{g} / \mathrm{ml})$ and $72 \mathrm{~h}$ after $\gamma$-irradiation (IRR; $5 \mathrm{~Gy})$. (b) The number of dead cells $24 \mathrm{~h}$ after ETO $(50 \mu \mathrm{g} / \mathrm{ml})$ treatment was determined by FACS. Cells were incubated 5 min with propidium iodide $(1 \mu \mathrm{g} / \mathrm{ml})$ and analyzed by FACS. (c) The mitochondrial membrane potential was determined by FACS after ETO $(50 \mu \mathrm{g} / \mathrm{ml})$ treatment at different time points. Cells were incubated 15 min with JC-1 probe and analyzed by FACS. (d and e) Caspase 3 activation after $24 \mathrm{~h}$ ETO $(50 \mu \mathrm{g} / \mathrm{ml})$ exposure was determined with DEVDase activity assay, respectively, in wild-type and mutant IDH1overexpressing LN18 and T98 cells. Results are expressed relative to wild-type IDH1-expressing cells. Results are expressed as the mean \pm S.E.M. of three experiments performed in triplicate. V, empty vector expressing cells; IDH1, wild-type IDH1-expressing cells; R132, IDH1 ${ }^{\mathrm{R} 132}$-expressing cells transfected. ${ }^{*} P<0.05$, ${ }^{* \star} P<0.01$ and ${ }^{* * *} P<0.001$

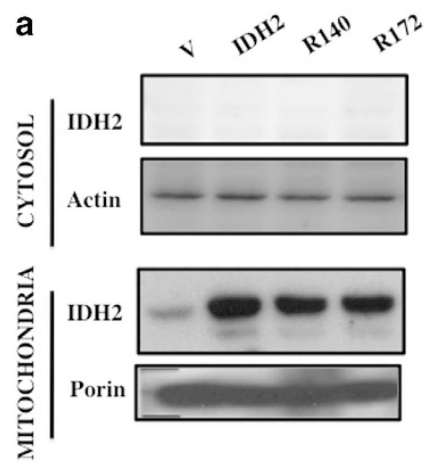

b

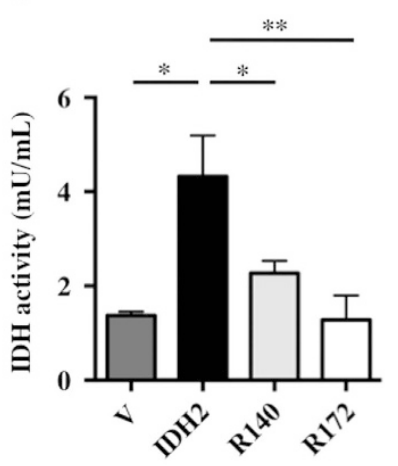

C

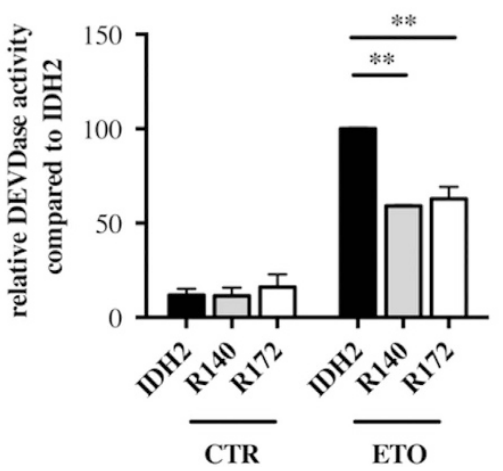

d

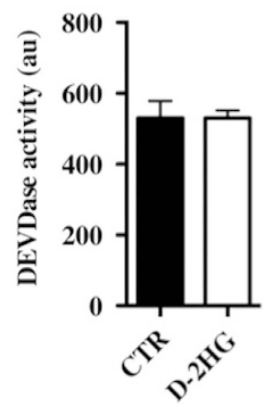

Figure 3 (a) Expression of wild-type (IDH2) or mutant IDH2 (R140 and R172) isoforms in stable overexpressing U251 cells was confirmed by immunoblotting using whole lysates $(40 \mu \mathrm{g})$ or purified mitochondria. Actin and porin were used as a loading control, respectively, for whole lysates and mitochondria. (b) IDH activity in cells overexpressing wild-type or mutant IDH2 isoforms. Cells $\left(1 \times 10^{6}\right)$ were plated, lysed the next day and subsequently assayed for their ability to generate NADPH. (c) Caspase 3 activation was determined with DEVDase activity assay in stable U251 cells expressing wild-type and mutant IDH2 isoforms after $24 \mathrm{~h} \mathrm{ETO} \mathrm{(50} \mu \mathrm{g} / \mathrm{ml})$ exposure. Results are expressed relative to wild-type IDH2-expressing cells. (d) Caspase 3 activation was determined with DEVDase activity assay after $24 \mathrm{~h} \mathrm{ETO} \mathrm{(50} \mu \mathrm{g} / \mathrm{ml})$ exposure in U251 cells treated with D-2HG $(3 \mathrm{mM})$ for 6 days. Results are expressed as the mean \pm S.E.M. of three experiments performed in triplicate. V, empty vector expressing cells; IDH2, wild-type IDH2-expressing cells; R140, IDH1 ${ }^{\mathrm{R} 140}$-expressing cells transfected; R172, IDH1 ${ }^{\mathrm{R} 172}$-expressing cells transfected. ${ }^{*} P<0.05,{ }^{\star \star} P<0.01$ and ${ }^{* \star *} P<0.001$

apoptosis escape, ${ }^{15}$ expression of these proteins, as well as BcIXL, XIAP, truncated BID (tBID) and survivin, was analyzed by western blot (Figure 4a). However, neither IDH1 ${ }^{\mathrm{R} 132}$ nor wild-type IDH1 expression affected protein expression of either control or ETO-treated cells. Expression of Bax and $\mathrm{Bcl} 2$ increased upon ETO treatment but to the same extent in vector, IDH1- and IDH1 ${ }^{\mathrm{R} 132}$-expressing cells. Of note, a similar pattern of expression of $\mathrm{Bax}$ and $\mathrm{Bcl} 2$ was observed in 

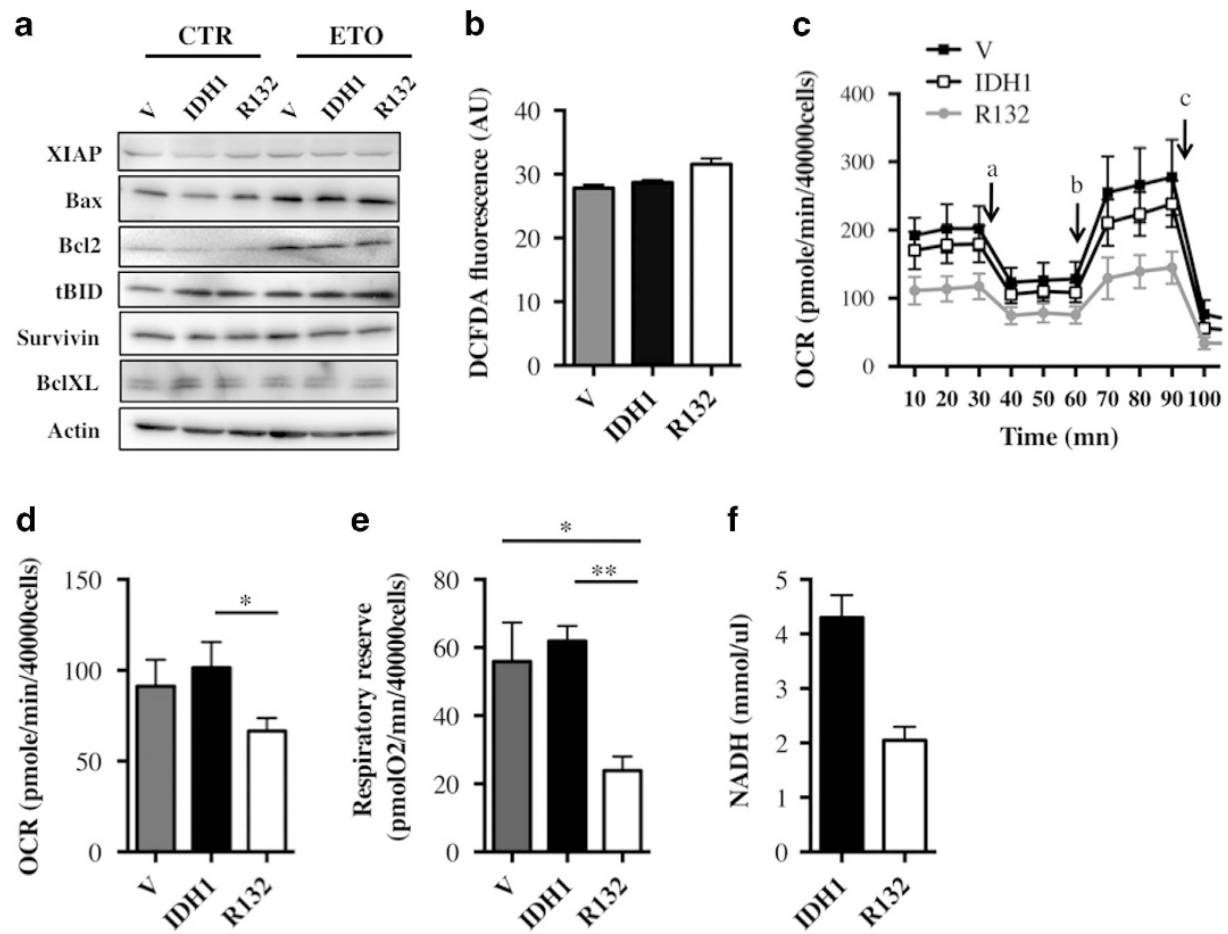

Figure 4 (a) Expression of proteins involved in apoptosis in U251 cells expressing empty vector, wild-type and mutant IDH1 isoforms. Whole lysates of cells were isolated $24 \mathrm{~h}$ after vehicule (V) or etoposide (ETO) treatment and analyzed (40 $\mu \mathrm{g}$ ) by immunoblotting with the indicated antibodies (left panel). Actin was used as a loading control. (b) ROS production was measured using the DCFDA probe. U251 cells expressing empty vector, wild-type and mutant IDH1 isoforms were seeded at $2.5 \times 10^{4}$ cells then incubated with the DCFDA probe. Fluorescence was measured at $538 \mathrm{~nm}$ every $3 \mathrm{~min}$ for $75 \mathrm{~min}$ and the slope corresponding to ROS production was calculated. (c) Oxygen consumption rate $(\mathrm{OCR})$ of stable cells expressing empty vector, wild-type and mutant IDH1 isoforms was measured over time. Cells $\left(4 \times 10^{4}\right)$ were plated and OCR was measured $24 \mathrm{~h}$ later by a XF24 Analyzer (Seahorse Bioscience). Mitochondrial inhibitors (oligomycin(a), CCCP(b), and rotenone and antimycin A(c)) were added as indicated with arrows. (d) Basal oxygen consumption was determined by measuring OCR as in c removing the non-mitochondrial oxygen consumption (OCR upon rotenone and antimycin A treatment). (e) The respiratory reserve of U251 cells expressing empty vector, wild-type and mutant IDH1 isoforms was measured by a XF24 Analyzer (Seahorse Bioscience). The respiratory reserve was determined as the difference between maximal OCR and basal OCR. (f) NADH production of cells expressing wild-type or mutant IDH1 isoforms. Cells $\left(1 \times 10^{6}\right)$ were plated, lysed the next day and subsequently assayed for their ability to produce NADH. Results are expressed as the mean \pm S.E.M. of three experiments performed in triplicate

cells treated with aKG or D-2HG before ETO (Supplementary Figure S1). As ROS and mitochondria have an important role in apoptosis, we then measured ROS production using the DCFDA probe and cell metabolism using the XF24 analyzer. Cellular ROS were not affected by either wild-type or $\mathrm{IDH}_{1}{ }^{\mathrm{R} 132}$ overexpression as shown in Figure $4 \mathrm{~b}$. Of note, ROS production was increased with rotenone, an inhibitor of the mitochondrial complex I able to induce mitochondrial ROS production, and decreased with EGCG, a polyphenol compound known for its antioxidant effect (Supplementary Figure S2). Interestingly, overexpression of $\mathrm{IDH} 1^{\mathrm{R} 132}$ and $\mathrm{IDH} 2^{\mathrm{R} 172}$ was associated with a slight but significant decreased in mitochondrial oxygen consumption rate (OCR) (Figures 4c and d, Supplementary Figure S3A). In addition to the basal OCR, maximal consumption rate, spare capacity and coupling efficiency were calculated from the recordings of the OCR following addition of different mitochondrial inhibitors (Figure 4c). Oligomycin is an inhibitor of ATP synthase and can be used to determine mitochondrial coupling efficiency, the efficiency with which mitochondria convert oxygen into ATP. CCCP, an uncoupler of mitochondrial oxidative phosphorylation raises OCR to its maximal rate, which allows the calculation of the respiratory reserve. Finally, rotenone and antimycin $A$, respectively, inhibits the complex I and complex III, which allows the determination of the non-mitochondrial oxygen consumption. Interestingly, a reduced respiratory reserve was observed in cells overexpressing IDH1 ${ }^{\mathrm{R} 132}$ (Figure 4e) or $\mathrm{IDH} 2^{\mathrm{R} 172}$ (Supplementary Figure S3B). To determine which complex was involved in the decreased respiratory reserve, OCR was recorded after sequential addition of rotenone and antimycin A. Complex I activity (left panel) contributed for $90 \%$ of total OCR, whereas complex II (right panel) contributed for only $10 \%$ (Supplementary Figure S4). Mutant IDH did not change the respective contribution of these complexes to mitochondrial respiration. Taken into account that the majority of the electrons entering the electron transport chain (ETC) are doing so at the level of complex I through oxidation of $\mathrm{NADH}$, the decreased mitochondrial respiratory reserve triggered by mutant IDH reflects probably a reduction of the NADH mitochondrial pool. In order to confirm this hypothesis, NADH level was measured in our cells (Figure 4f). Indeed, NADH level was decreased in IDH1 ${ }^{\mathrm{R} 132}$ cells compared with IDH1 cells.

No difference was observed in the coupling efficiency between ETC activity and ATP synthesis or in glycolysis as reflected by the extracellular acidification rate (ECAR; Supplementary Figure S5). We then assessed the mitochondrial OCR of cells treated with aKG or D-2HG for 6 days. The addition of $a \mathrm{KG}$ significantly increased both 
the basal and the respiratory reserve, which is not surprising as this metabolite directly fuels the TCA cycle (Figures $5 a$ and b). However, D-2HG affected neither the basal OCR nor the respiratory reserve. Glycolysis was not affected by D-2HG (data not shown).

Oxamate prevents reduction of both the respiratory reserve and ETO-induced apoptosis triggered by $\mathrm{IDH} 1^{\mathrm{R} 132}$. Oxamate is a known inhibitor of lactate dehydrogenase. In order to determine whether forcing the cell into oxidative phosphorylation could affect the sensitivity to apoptosis after ETO treatment, cells were exposed to oxamate $(3 \mathrm{mM}$ for 3 days) before metabolic analysis or ETO treatment. Surprisingly, lactate dehydrogenase (LDH) activity was not inhibited by oxamate as shown in Figures $6 \mathrm{a}$ and $\mathrm{b}$. Furthermore, although oxamate did not affect basal OCR or the respiratory reserve of vector and IDH1-overexpressing cells, the reduction in both basal OCR and the respiratory reserve observed in overexpressing IDH1 ${ }^{\mathrm{R} 132}$ cells was restored by oxamate (Figures $6 \mathrm{c}$ and d). ETO-induced apoptosis was then evaluated in the
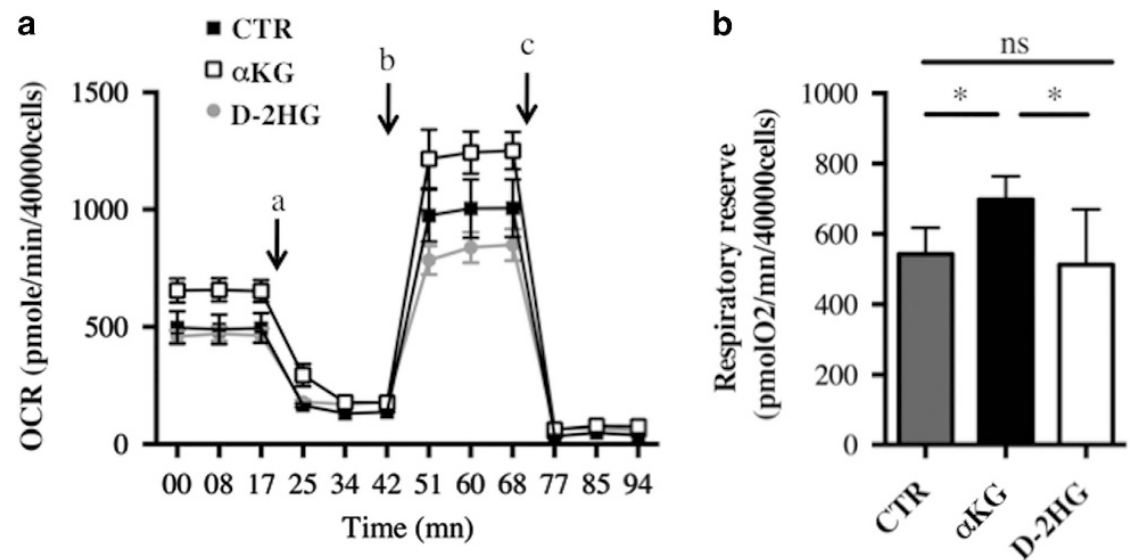

Figure $5 \mathrm{OCR}(\mathrm{a})$ and mitochondrial respiratory reserve (b) were measured respectively as in Figure 4 in cells treated or not with $5 \mathrm{mM} \alpha \mathrm{KG}$ or D-2HG for 5 days. Results are expressed as the mean \pm S.E.M. of three experiments performed in triplicate. ${ }^{*} P<0.05,{ }^{\star \star} P<0.01$ and ${ }^{* \star *} P<0.001$

a
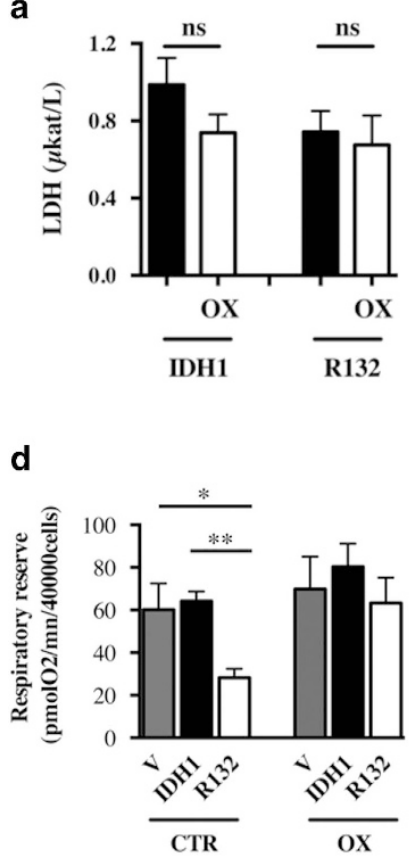
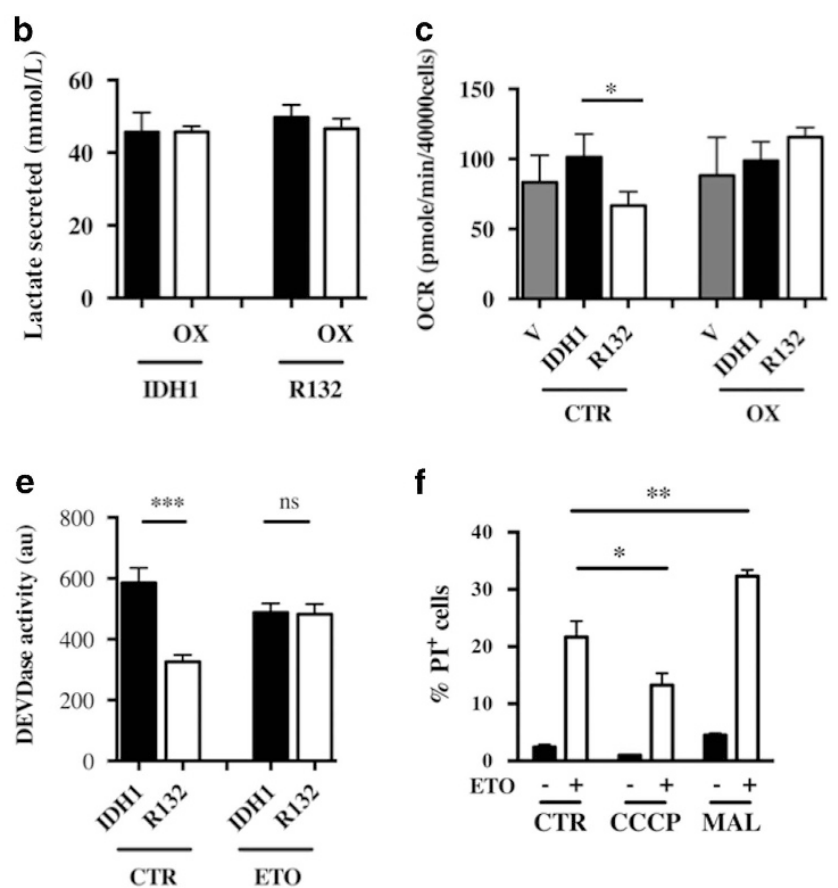

f

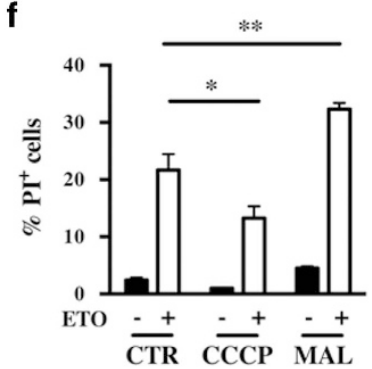

Figure 6 Lactate deshydrogenase activity (a) and lactate production (b) of U251 cells expressing IDH1 isoforms. Cells were treated or not with $3 \mathrm{mM}$ oxamate (OX) for $48 \mathrm{~h}$. (c) Basal oxygen consumption was determined by measuring OCR as in Figure 4c in cells expressing IDH1 isoforms treated or not with $3 \mathrm{mM}$ oxamate for 7 days. (d) Mitochondrial respiratory reserve was determined as in Figure $4 \mathrm{e}$ in cells treated or not with $3 \mathrm{mM}$ oxamate for 7 days. (e) Caspase 3 activation was determined using a DEVDase activity assay $24 \mathrm{~h}$ after ETO-induced apoptosis in U251 cells treated or not for 7 days with $3 \mathrm{mM}$ oxamate. (f) The number of dead cells was measured $24 \mathrm{~h}$ after a concomitant treatment with CCCP $(1 \mu \mathrm{M})$ or malate $(3 \mathrm{mM})$ and ETO $(50 \mu \mathrm{g} / \mathrm{ml})$. The number of dead cells was determined by FACS after propidium iodide $(1 \mu \mathrm{g} / \mathrm{ml})$ staining. Results are expressed as the mean \pm S.E.M. of three experiments performed in triplicate 
Wild-type IDHI overexpressing cells

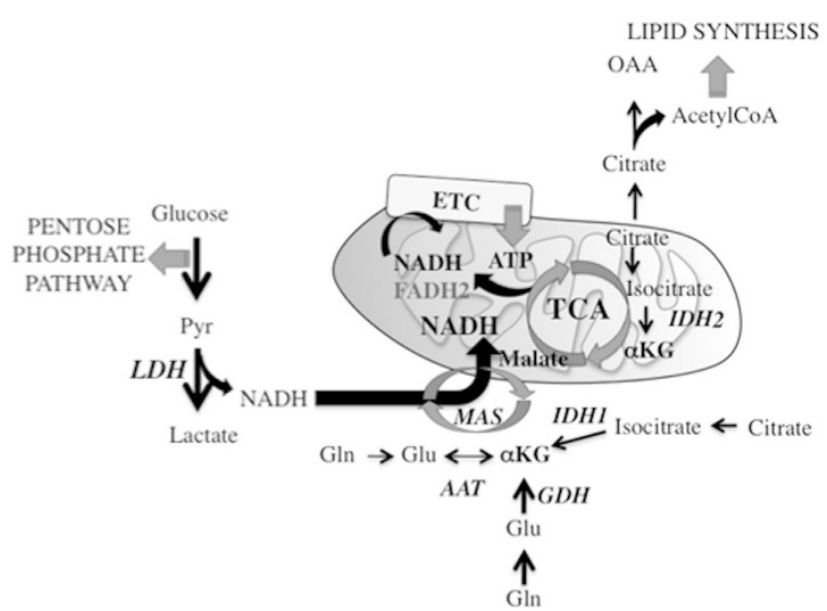

IDHI ${ }^{R 132}$ overexpressing cells

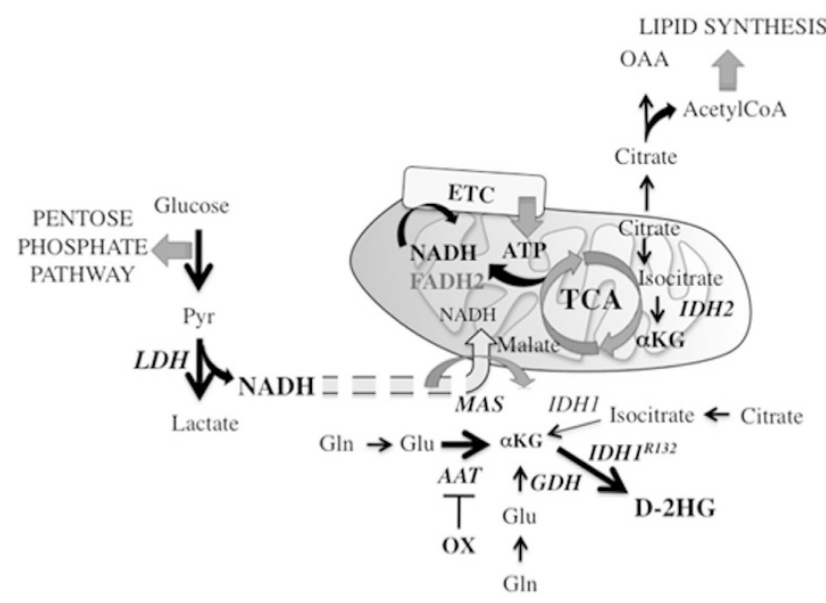

Figure 7 Metabolic alterations driven by IDH mutation in U251 cells. In cancer cells, glycolysis upregulation generates the production of reducing equivalents NADH, which is then shuttles from the cytosol to mitochondria with the MAS. Glutamine is converted to glutamate, which is further converted to $\alpha \mathrm{KG}$ by GDH. IDH also produces $\alpha \mathrm{KG}$ from isocitrate. In cells expressing IDH1 ${ }^{\mathrm{R} 132}$, IDH1 ${ }^{\mathrm{R} 132}$ converts $\alpha \mathrm{KG}$ into $\mathrm{D}-2 \mathrm{HG}$. To limit cellular $\alpha \mathrm{KG}$ depletion, IDH1 ${ }^{\mathrm{R} 132}$-overexpressing cells diverts AAT from MAS function to produce $\alpha \mathrm{KG}$. As a result, glycolytic NADH is no longer fully imported into mitochondria. AAT, aspartate aminotransferase; ETC, electron transport chain; GDH, glutamate dehydrogenase; Gln, Glutamine; Glu, Glutamate; IDH, isocitrate dehydrogenase; LDH, lactate dehydrogenase; MAS, malate-aspartate shuttle; Pyr, pyruvate; TCA, tricarboxylic acid cycle

presence of oxamate. Interestingly, caspase-3 activation was not modified with oxamate after ETO treatment in IDH1-overexpressing cells (Figure 6e). However, the sensitivity of $\mathrm{IDH} 1^{\mathrm{R} 132}$ cells to ETO in the presence of oxamate was comparable to that of IDH1 cells (Figure 6e). Altogether, these results strongly suggest that resistance to ETO-induced apoptosis is associated with a depleted mitochondrial NADH pool triggered by mutant IDH expression. In order to determine whether the mitochondrial NADH level can affect cell sensitivity to ETO, U251 cells were treated concomitantly with either CCCP $(1 \mu \mathrm{M})$ or malate $(5 \mathrm{mM})$ and ETO and cell death was analyzed $24 \mathrm{~h}$ later. Indeed, although addition of CCCP was associated with reduced cell death, addition of malate led to increased cell death (Figure 6f).

\section{Discussion}

One of the main consequences triggered by tumor-associated IDH mutations is D-2HG accumulation as mutant IDH leads to the synthesis of $\mathrm{D}-2 \mathrm{HG}$ instead of $a \mathrm{KG}^{9}{ }^{9}$ In this paper, we show that mutant IDH overexpression increases cell proliferation of glioma cells and reduces ETO-induced apoptosis. Although the metabolite $\mathrm{D}-2 \mathrm{HG}$ is involved in the proliferation phenotype, its potential role is excluded for the latter phenotype on cell death. This is the first study showing that D-2HG does not fully recapitulate the IDH mutation phenotype.

The oncogenic potential of D-2HG has been extensively studied since the discovery of IDH mutations. In fact, D-2HG and $a K G$ are structurally similar, suggesting that D-2HG may act as a competitive inhibitor of a number of aKGdioxygenases. This hypothesis has been confirmed in vitro as the addition of D-2HG to cells causes the inhibition of multiple aKG-dioxygenases. ${ }^{14}$ Epigenetic profiling of $A M L$ and glioma patient cohorts show that IDH mutations trigger a global DNA hypermethylation. ${ }^{16,17}$ Several studies have shown that D-2HG is sufficient to disrupt TET2 function and impair histone demethylation. ${ }^{11,13,18}$ In addition, this metabolite impacts a number of cellular processes such as increasing cell proliferation and blocking cell differentiation. ${ }^{18,19}$ In agreement with these reports, we show an increase in cell proliferation at both low and high densities with either mutant IDH or D-2HG treatment. However, other groups ${ }^{20}$ have shown reduced growth with overexpression mutant IDH, generally associated with increased oxidative stress. These data suggest that metabolic abnormalities associated with mutant IDH are cell type specific.

IDH mutations cause many other metabolic abnormalities besides D-2HG accumulation. ${ }^{21}$ Cells expressing mutant IDH exhibit decreased NADPH because of the lack of conversion of isocitrate to aKG and also because of the consumption of NADPH in the conversion of $a K G$ to D-2HG. NADPH serves as an electron carrier for the maintenance of redox homeostasis and reductive biosynthesis with separate cytosolic and mitochondrial pools. Thus, mutant IDH may lead to increased intracellular ROS oxidation. However, in our cell model, we show that mutant IDH does not affect the total cellular ROS nor affect superoxide dismutase 2 and catalase expression (data not shown). Studies by Leonardi et al. ${ }^{11}$ have indicated that mutant IDH may compromise the ability of this enzyme to catalyze the Gln-dependent reductive carboxylation reaction. This pathway can be stimulated by a perturbation in the redox ratio. However, our mutant IDH cells show no selective sensitivity to Gln metabolism inhibitors such as EGCG and BPTES (Supplementary Figure S6). In our model, oxidative stress is not increased with the overexpression of mutants IDH, which could explain why there was no increase in GIn dependency in our glioma cell lines.

Several studies have been published showing opposite effects of mutant IDH on cell death. We show that mutant IDH increases the resistance of gliomas to specific cell death 
stimuli. Our results are in agreement with the data published by the group of Park, showing an increased sensitivity to radiation in different cell types when IDH1 or IDH2 is silenced. ${ }^{22-25}$ However, other studies show increased cell sensitivity to cell death when mutant IDH is overexpressed, in particular in ROS-mediated cell death such as radiation- ${ }^{26}$ or BCNU-induced cell death. ${ }^{27}$ However, these cells are addicted to glutaminolysis contrary to the glioma cell lines used in our study. This difference in metabolism could explain the discrepancy observed in cell death sensitivity between the different cell lines. Another explanation for these opposing results could be the specific genetic background of the different cell types, in particular the expressed forms of p53 and EGFR, the methylation status of MGMT and the presence or not of PTEN. This raises the possibility that, in general, the phenotype associated with mutant IDH may be restricted to some cell lines. Mutant IDH may also increase the resistance of gliomas to specific cell death stimuli. Resistance to apoptosis by $\mathrm{IDH} 1^{\mathrm{R} 132}$ provides a rationale for the high frequency of $\mathrm{IDH} 1^{\mathrm{R} 132}$ in secondary GBM.

Interestingly, our data show that both mutant IDH1 and mutant IDH2 that, respectively, are expressed in cytosol and mitochondria, lead to decreased mitochondrial respiratory reserve. Numerous studies have shown that IDH mutants use $a \mathrm{KG}$ as a substrate to produce D-2HG instead of producing it. This drain of $a K G$ must be balanced to some extent by the cells. There are two ways of converting glutamate (Glu) into aKG, either by deamination through glutamate dehydrogenase $(\mathrm{GDH})$ or by transamination through aspartate aminotransferase (AAT). ${ }^{28}$ Usually, AAT functions in tandem with the malate dehydrogenase (MDH) in the malate-aspartate shuttle (MAS), which transfers reducing equivalent $\mathrm{NADH}$ from the cytosol to mitochondria. Glycolysis, which is highly upregulated in cancer cells, is a key source of the reduced form of cytosolic $\mathrm{NADH}$, mainly at the level of $\mathrm{LDH}^{29}$ As a result, the activity of MAS is increased to shuttle the glycolytic NADH into mitochondria. ${ }^{30}$ However, it has also been shown that the presence or the lack of mitochondrial substrates could greatly influence the ability of AAT to effectively compete with GDH for Glu. ${ }^{31}$ We speculate that to limit cytosolic and mitochondrial aKG depletion induced by the presence of mutant IDH, cells are using AAT, independently of $\mathrm{MDH}$, to produce $a \mathrm{KG}$ rather than to shuttle $\mathrm{NADH}$ in mitochondria (Figure 7). As a result, the dissociation of AAT activity from $\mathrm{MDH}$ results in the accumulation of the reductive power of malate trapped in the cytosol and the reduction of mitochondrial NADH pool built from the MAS activity. Altogether, these effects lead ultimately to a reduced mitochondrial respiratory reserve. Further experiments such as direct inhibition of AAT need to be performed in order to confirm our hypothesis. However, it is in agreement with metabolomic studies showing that mutant IDH is associated with decreased fumarate and malate levels, as well as mitochondrial dysfunction. ${ }^{32}$ Furthermore, it is reinforced with the recovery of the mitochondrial respiratory reserve with oxamate. Indeed, besides being an inhibitor of $\mathrm{LDH}$, oxamate also inhibits AAT. ${ }^{33,34}$ In our study, oxamate did not decrease lactate secretion as predicted by its LDH inhibitory action but instead prevented the mitochondrial respiratory reserve loss only in mutant IDH-overexpressing cells.

This metabolic alteration has an important role in the resistance to ETO-induced apoptosis in IDH ${ }^{\mathrm{R} 132}$-overexpressing cells. ETO is a DNA-topoisomerase II inhibitor widely used in the treatment of diverse tumors. ${ }^{35}$ Like many other agents, ETO can be used in monotherapy or in combination with other treatments. One of the major pathways of ETO metabolism in cells involves cytochrome P450 reductase, which uses NADPH as a cofactor for its enzymatic activity. Furthermore, it has been shown that ETO-induced apoptosis requires fully functional mitochondria. ${ }^{36}$ Our data and others show that mutant IDH decreases the cellular NADPH pool combined with the altered mitochondrial metabolism ${ }^{37}$ may explain the specific resistance to ETO of IDH1 ${ }^{\mathrm{R} 132}$-overexpressing cells.

Collectively, our data support the hypothesis that mutant IDH affects tumor progression and therapy resistance, which may, at least in part, explain the high frequency of IDH mutation in secondary GBM. On the basis of our and other observations, D-2HG mediates pro-tumorigenic effects, whereas altered metabolism and in particular the imbalance of NAD+ and NADP+ coenzymes are associated with cell resistance to specific cell death stimuli.

In conclusion, IDH mutation deregulates mitochondrial metabolism and as a consequence alters cell sensitivity to specific stimuli. Clinically, a better understanding of IDH mutations will enable IDH-directed therapies to be developed in the future.

\begin{abstract}
Materials and Methods
Cell culture and stable transfection. Glioblastoma U251 and LN18 cell lines were cultured at $37^{\circ} \mathrm{C}$ in a humidified atmosphere with $5 \% \quad \mathrm{CO}_{2}$ in Dulbecco's modified Eagle's medium (DMEM) containing $5 \mathrm{~g} / \mathrm{l}$ glucose and supplemented with $100 \mathrm{U} / \mathrm{ml}$ penicillin, $100 \mu \mathrm{g} / \mathrm{ml}$ streptomycin, $2 \mathrm{mM} \mathrm{Gln}$ and, respectively, $10 \%$ and $5 \%$ fetal calf serum (FCS). T98 cells were cultured in DMEM $1 \mathrm{~g} / \mathrm{l}$ glucose, supplemented with penicillin, streptomycin, Gln and $10 \%$ FCS. Cells were seeded in 12-well plates and transfected with Lipofectamine 2000 transfection reagent as recommended by the manufacturer (Life Technologies, Carlsbad, CA, USA). The expression vectors for wild-type and mutated IDH1 and IDH2 were described previously. ${ }^{38}$ U251, LN18 and T98 stable cells lines overexpressing IDH were selected using geneticin (Life Technologies). Hydroxyglutarate was purchased from Peptech (Bedford, MA, USA) and $\alpha \mathrm{KG}$, dimethyl- $\alpha \mathrm{KG}$ and oxamate from Sigma (St Louis, MO, USA). For cell death experiments, $0.5 \times 10^{6}$ cells were plated and treated the next day with $50 \mu \mathrm{g} / \mathrm{ml}$ ETO (Mylan, St Priest, France), $50 \mathrm{ng} / \mathrm{ml}$ TRAIL (PreproTech, Neuilly sur-Seine, France), $60 \mathrm{ng} / \mathrm{ml}$ FASL (PreproTech) and $15 \mu \mathrm{g} / \mathrm{ml}$ cisplatin (Mylan). $\gamma$-Irradiation was carried out in a Faxitron CP160 irradiator (Faxitron X-ray Corporation, Tucson, AZ, USA) at a dose rate of 5 Gy. The number of dead cells was evaluated by FACS after incubation of $5 \mathrm{~min}$ with propidium iodide (Sigma).
\end{abstract}

Cell counts, viability and clonogenicity assay. Cell counts and viability were performed using the Countess optics and image automated cell counter (Life Technologies). Cells were mixed with trypan blue (50/50) and loaded into a Countess chamber slide. The image analysis software was used to automatically analyze the acquired cell images from the sample to give cell count and viability. Data were plotted either as the number of viable cells for cell proliferation or as the percent of dead cells for viability assessment. For cell proliferation, cells were seeded at 100.000 cells and counted every 3 to 4 days. For the clonogenicity assay, 500 cells were seeded on a 6-well plate. After 1 week, cells were fixed and stained with a solution of violet crystal $0.05 \%$ in ethanol $50 \%$. The number of colonies is counted. 
ROS and mitochondrial membrane potential. ROS production was measured using a DCFDA probe (Life Technologies). Cells were seeded at $2 . \times 10^{4}$ cells in a 96-well plate and incubated with the DCFDA probe. The fluorescence is measured at $538 \mathrm{~nm}$ every $3 \mathrm{~min}$ for $75 \mathrm{~min}$. Mitochondrial membrane potential was evaluated by FACS analysis using JC-1 probe (Life Technologies) staining accordingly to the manufacturer's instruction.

Biochemical analysis. Production of NADH and NADPH was measured using, respectively, the NAD/NADH kit (Abcam, Cambridge, UK) and the NADP/NADPH kit (Abcam) according to the manufacturer's instruction. Lactate and LDH activity were measured using the Roche diagnostic kit on a Cobas 8000 (Roche Diagnostics, Mannheim, DE, USA) as described previously. ${ }^{39}$

Protein lysates, immunoblotting and caspase activation. Cells were lysed at the indicated time points in RIPA lysis buffer $(25 \mathrm{mM}$ Tris- $\mathrm{HCl}, \mathrm{pH}$ 7.6 containing $150 \mathrm{mM} \mathrm{NaCl}, 1 \% \mathrm{NP} 40,1 \% \mathrm{Na}$-deoxycholate and $0.1 \%$ SDS) supplemented with protease inhibitors. Protein concentration was determined using BCA protein assay (Sigma). Samples were adjusted accordingly, analyzed by SDS-PAGE and subsequent immunoblotting with antibodies that recognize IDH1 (Abcam), IDH2 (Abcam), IDH1-R132H (Dianova, Hamburg, Germany), actin (Millipore, Billerica, MA, USA), Bax (BD Pharmingen, San Jose, CA, USA), Bcl2 (BD Pharmingen), XIAP (R\&D system, Abingdon, UK), tBid (R\&D System), porin (Calbiochem, Nottingham, UK), survivin (Cell Signaling Technology, Danvers, MA, USA). HRP-conjugated secondary antibodies were from BioRad (Marnes-laCoquette, France). The ImageJ 1.42q software (NIH) was used to digitally quantify the signal intensities of western blot bands. Caspase 3 activity was quantified using the fluorogenic substrate Ac-DEVD-AMC, as described in. ${ }^{15}$

OCR and extracellular consumption rate (ECAR). Cells were plated at 40000 cells per well in 24-well XF (extracellular flux) cell culture microplate (Seahorse Bioscience, Copenhagen, Denmark). OCR was measured the next day using a XF24 Analyzer (Seahorse Bioscience). Cells were equilibrated for $1 \mathrm{~h}$ at $37^{\circ} \mathrm{C}$ in bicarbonate-free DMEM (Sigma) supplemented with $25 \mathrm{mM}$ glucose, $1 \mathrm{mM}$ pyruvate and $2 \mathrm{mM}$ Gln, $\mathrm{pH} 7.3$ before any measurement. To determine mitochondrial parameters, OCR was measured at baseline and after addition of oligomycin $(0.4 \mu \mathrm{M})$, FCCP $(2 \mu \mathrm{M})$, rotenone $(0.6 \mu \mathrm{M})$ and antimycin $\mathrm{A}(0.6 \mu \mathrm{M})$. All measurements were done at least in three wells per condition, twice for each experiment. Respiratory reserve was calculated as maximal OCR, after oligomycin and CCCP injections, minus basal OCR. Coupling efficiency corresponded to OCR inhibition by oligomycin. Relative contribution of complex I and II to OCR corresponded, respectively, to OCR inhibition by rotenone and antimycin $\mathrm{A}$.

Statistical analysis. Experiments were done at least three times and data were analyzed using GraphPad Prism 5.00 (GraphPad Software, San Diego, CA, USA). Differences with $P$-values $<0.05$ were considered statistically significant.

\section{Conflict of Interest}

The authors declare no conflict of interest.

Acknowledgements. This work was supported by grants from 'La Ligue contre le Cancer' and 'Region-Pays de la Loire'. K Oizel was funded by Region-Pays de la Loire. We thank CB Thompson for providing PCMV-IDH1 and PCMV-IDH1 ${ }^{\mathrm{R} 132}$ plasmids.

1. Parsons DW, Jones S, Zhang X, Lin JC-H, Leary RJ, Angenendt $P$ et al. An integrated genomic analysis of human glioblastoma multiforme. Science 2008; 321: 1807-1812.

2. Yan H, Parsons DW, Jin G, McLendon R, Rasheed BA, Yuan W et al. IDH1 and IDH2 mutations in gliomas. N Engl J Med 2009; 360: 765-773.

3. Mardis ER, Ding L, Dooling DJ, Larson DE, McLellan MD, Chen $\mathrm{K}$ et al. Recurring mutations found by sequencing an acute myeloid leukemia genome. $N$ Engl J Med 2009; 361: 1058-1066.

4. Amary MF, Bacsi K, Maggiani F, Damato S, Halai D, Berisha F et al. IDH1 and IDH2 mutations are frequent events in central chondrosarcoma and central and periosteal chondromas but not in other mesenchymal tumours. J Pathol 2011; 224: 334-343.

5. Louis DN, Ohgaki H, Wiestler OD, Cavenee WK, Burger PC, Jouvet A et al. The 2007 WHO Classification of Tumours of the Central Nervous System. Acta Neuropathol (Berl) 2007; 114: 97-109.

6. Abdel-Wahab O, Patel J, Levine RL. Clinical implications of novel mutations in epigenetic modifiers in AML. Hematol Oncol Clin North Am 2011; 25: 1119-1133.

7. Rakheja D, Konoplev S, Medeiros LJ, Chen W. IDH mutations in acute myeloid leukemia. Hum Pathol 2012; 43: 1541-1551.

8. Pecqueur C, Oliver L, Oizel K, Lalier L, Vallette FM. Targeting metabolism to induce cell death in cancer cells and cancer stem cells. Int J Cell Biol 2013; 2013: 1-13.

9. Dang L, White DW, Gross S, Bennett BD, Bittinger MA, Driggers EM et al. Cancer-associated IDH1 mutations produce 2-hydroxyglutarate. Nature 2009; 462: 739-744.

10. Reitman ZJ, Yan $\mathrm{H}$. Isocitrate dehydrogenase 1 and 2 mutations in cancer: alterations at a crossroads of cellular metabolism. J Natl Cancer Inst 2010; 102: 932-941.

11. Leonardi R, Subramanian C, Jackowski S, Rock CO. Cancer-associated isocitrate dehydrogenase mutations inactivate NADPH-dependent reductive carboxylation. J Biol Chem 2012; 287: 14615-14620.

12. Oermann EK, Wu J, Guan K-L, Xiong Y. Alterations of metabolic genes and metabolites in cancer. Semin Cell Dev Biol 2012; 23: 370-380.

13. Chowdhury R, Yeoh KK, Tian Y-M, Hillringhaus L, Bagg EA, Rose NR et al. The oncometabolite 2-hydroxyglutarate inhibits histone lysine demethylases. EMBO Rep 2011; 12: 463-469.

14. Xu W, Yang H, Liu Y, Yang Y, Wang P, Kim S-H et al. Oncometabolite 2-hydroxyglutarate is a competitive inhibitor of $\alpha$-ketoglutarate-dependent dioxygenases. Cancer Cell 2011; 19: $17-30$.

15. Cartron P-F, Juin P, Oliver L, Martin S, Meflah K, Vallette FM. Nonredundant role of bax and bak in bid-mediated apoptosis. Mol Cell Biol 2003; 23: 4701-4712.

16. Figueroa ME, Abdel-Wahab O, Lu C, Ward PS, Patel J, Shih A et al. Leukemic IDH1 and IDH2 mutations result in a hypermethylation phenotype, disrupt TET2 function, and impair hematopoietic differentiation. Cancer Cell 2010; 18: 553-567.

17. Turcan S, Rohle D, Goenka A, Walsh LA, Fang F, Yilmaz E et al. IDH1 mutation is sufficient to establish the glioma hypermethylator phenotype. Nature 2012; 483: 479-483.

18. Lu C, Ward PS, Kapoor GS, Rohle D, Turcan S, Abdel-Wahab O et al. IDH mutation impairs histone demethylation and results in a block to cell differentiation. Nature 2012; 483: 474-478.

19. Losman J-A, Looper RE, Koivunen P, Lee S, Schneider RK, McMahon C et al. (R)-2hydroxyglutarate is sufficient to promote leukemogenesis and its effects are reversible. Science 2013; 339: 1621-1625.

20. Bralten LBC, Kloosterhof NK, Balvers R, Sacchetti A, Lapre L, Lamfers M et al. IDH1 $\mathrm{R} 132 \mathrm{H}$ decreases proliferation of glioma cell lines in vitro and in vivo. Ann Neurol 2011; 69: $455-463$

21. Reitman ZJ, Duncan CG, Poteet E, Winters A, Yan L-J, Gooden DM et al. Cancer-associated isocitrate dehydrogenase 1 (IDH1) $\mathrm{R} 132 \mathrm{H}$ mutation and D-2-hydroxyglutarate stimulate glutamine metabolism under hypoxia. J Biol Chem 2014; 22: 23318-23328

22. Kim SY, Yoo YH, Park J-W. Silencing of mitochondrial NADP(+)-dependent isocitrate dehydrogenase gene enhances glioma radiosensitivity. Biochem Biophys Res Commun 2013; 433: 260-265.

23. Jung KH, Park J-W. Suppression of mitochondrial NADP(+)-dependent isocitrate dehydrogenase activity enhances curcumin-induced apoptosis in HCT116 cells. Free Radic Res 2011; 45: 431-438.

24. Kil IS, Jung KH, Nam WS, Park J-W. Attenuated mitochondrial NADP+-dependent isocitrate dehydrogenase activity enhances EGCG-induced apoptosis. Biochimie 2011; 93: $1808-1815$

25. Kil IS, Chung KH, Park J-W. Silencing of mitochondrial NADP+-dependent isocitrate dehydrogenase gene enhances selenite-induced apoptosis. Free Radic Res 2010; 44: 332-339.

26. Li S, Chou AP, Chen W, Chen R, Deng Y, Phillips HS et al. Overexpression of isocitrate dehydrogenase mutant proteins renders glioma cells more sensitive to radiation. Neuro Oncol 2013; 15: 57-68.

27. Mohrenz IV, Antonietti P, Pusch S, Capper D, Balss J, Voigt S et al. Isocitrate dehydrogenase 1 mutant $\mathrm{R} 132 \mathrm{H}$ sensitizes glioma cells to BCNUinduced oxidative stress and cell death. Apoptosis 2013; 18: 1416-1425.

28. McKenna MC, Stevenson JH, Huang X, Hopkins IB. Differential distribution of the enzymes glutamate dehydrogenase and aspartate aminotransferase in cortical synaptic mitochondria contributes to metabolic compartmentation in cortical synaptic terminals. Neurochem Int 2000; 37: 229-241.

29. Gatenby RA, Gillies RJ. Why do cancers have high aerobic glycolysis? Nat Rev Cancer 2004; 4: 891-899.

30. Greenhouse WV, Lehninger AL. Occurrence of the malate-aspartate shuttle in various tumor types. Cancer Res 1976; 36: 1392-1396.

31. Erecińska M, Nelson D. Activation of glutamate dehydrogenase by leucine and its nonmetabolizable analogue in rat brain synaptosomes. J Neurochem 1990; 54: 1335-1343.

32. Thornburg JM, Nelson KK, Clem BF, Lane AN, Arumugam S, Simmons A et al. Targeting aspartate aminotransferase in breast cancer. Breast Cancer Res 2008; 10: R84. 
33. Reitman ZJ, Jin G, Karoly ED, Spasojevic I, Yang J, Kinzler KW et al. Profiling the effects of isocitrate dehydrogenase 1 and 2 mutations on the cellular metabolome. Proc Natl Acad Sci USA 2011; 108: 3270-3275.

34. Rej R. Measurement of aspartate aminotransferase activity: effects of oxamate. Clin Chem 1979; 25: 555-559.

35. Hande KR. Etoposide: four decades of development of a topoisomerase II inhibitor. Eur J Cancer 1998; 34: 1514-1521.

36. Kulawiec M, Owens KM, Singh KK. Cancer cell mitochondria confer apoptosis resistance and promote metastasis. Cancer Biol Ther 2009; 8: 1378-1385.

37. Grassian AR, Parker SJ, Davidson SM, Divakaruni AS, Green CR, Zhang X et al. IDH1 mutations alter citric acid cycle metabolism and increase dependence on oxidative mitochondrial metabolism. Cancer Res 2014; 74: 3317-3331.

38. Ward PS, Patel J, Wise DR, Abdel-Wahab O, Bennett BD, Coller HA et al. The common feature of leukemia-associated IDH1 and IDH2 mutations is a neomorphic enzyme activity converting alpha-ketoglutarate to 2-hydroxyglutarate. Cancer Cell 2010; 17: $225-234$.
39. Gratas C, Séry Q, Rabé M, Oliver L, Vallette FM. Bak and Mcl-1 are essential for Temozolomide induced cell death in human glioma. Oncotarget 2014; 5: 2428-2435.

(c) (i) Cell Death and Disease is an open-access journal published by Nature Publishing Group. This work is licensed under a Creative Commons Attribution 4.0 International License. The images or other third party material in this article are included in the article's Creative Commons license, unless indicated otherwise in the credit line; if the material is not included under the Creative Commons license, users will need to obtain permission from the license holder to reproduce the material. To view a copy of this license, visit http://creativecommons.org/licenses/by/4.0/

Supplementary Information accompanies this paper on Cell Death and Disease website (http://www.nature.com/cddis) 